AsCLEPIO. Revista de Historia de la Medicina y de la Ciencia

66 (2), julio-diciembre 2014, p053

ISSN-L:0210-4466

http://dx.doi.org/10.3989/asclepio.2014.17

DOSSIER: BIOPODER Y DETERMINISMOS EN SUDAMÉRICA DURANTE EL SIGLO XX / DOSSIER: BIOPOWER AND DETERMINISM IN SOUTH AMERICA DURING THE TWENTIETH CENTURY

\title{
DE PLANTAS Y HOMBRES: CÓMO LOS GENETISTAS SE VINCULARON A LA EUGENESIA EN BRASIL (UN ESTUDIO DE CASO, 1929-1933)
}

\section{Paula Arantes Botelho Briglia Habib}

\author{
Fiocruz, Brasil \\ pbrigliahabib@uol.com.br

\section{Robert Wegner} \\ Fiocruz, Brasil \\ robertwegnercoc@gmail.com
}

Recibido: 7 julio 2013; Aceptado: 14 abril 2014.

Cómo citar este artículo/Citation: Habib, Paula Arantes Botelho Briglia y Robert Wegner (2014), "De plantas y hombres: cómo los genetistas se vincularon a la eugenesia en Brasil (un estudio de caso, 1929-1933)", Asclepio, 66 (2): p053. doi: http://dx.doi. org/10.3989/asclepio.2014.17

RESUMEN: En el presente artículo pretendemos reflexionar sobre la trayectoria de Octávio Domingues (1897-1972) y Salvador Toledo Piza Jr. (1898-1988), dos genetistas de una escuela agrícola del Estado de São Paulo que se vincularon al principal propagador de la eugenesia en Brasil, Renato Kehl (1889-1974). Ese acercamiento se concretó en su asociación alrededor de la publicación del Boletim de Eugenia, importante vehículo de divulgación del movimiento eugenésico entre los años 1929 y 1933. A partir del análisis de esa publicación periódica, ha sido posible detectar que, aunque los dos partían de la teoría mendeliana de la herencia, Toledo Piza Jr. consideraba el mestizaje de la población brasileña como algo que iba en contra de la naturaleza, mientras que Octávio Domingues la juzgaba saludable.

PALABRAS CLAVE: Genética; Eugenesia; Boletim de Eugenia; Historia de la Agricultura; Brasil.

OF PLANTS AND MEN: HOW GENETICISTS WERE LINKED TO EUGENICS IN BRAZIL (1929-1933)

ABSTRACT: The aim of this article is to analyse the trajectory of Octávio Domingues (1897-1972) and Salvador de Toledo Piza Jr. (1898-1988), two geneticists in the Agricultural School of São Paulo State, who engaged with the main propagandist for the eugenics in Brazil, Renato Kehl (1889-1974). This approach is embodied in its association surrounding the publication of the Boletim de Eugenia, an important vehicle for the dissemination of the eugenics movement between the years 1929 and 1933 . From the analysis of this journal, it was possible to detect that even sharing both Mendelian theory of heredity, Toledo Piza Jr. considered miscegenation of the Brazilian population as something against nature, while Octávio Domingues judged it healthy.

KEY WORDS: Genetics; Eugenics; Boletim de Eugenia; Agricultural History; Brazil.

Copyright: (c) 2014 CSIC. Este es un artículo de acceso abierto distribuido bajo los términos de la licencia Creative Commons Attribution-Non Commercial (by-nc) Spain 3.0. 


\section{INTRODUCCIÓN}

El presente artículo pretende investigar cómo se estableció una colaboración entre el médico Renato Kehl (1889-1974), el zootecnista Octávio Domingues (1897-1972) y el zoólogo Salvador Toledo Piza Jr. (1898-1988), los dos últimos profesores en la Escuela Superior de Agricultura «Luiz de Queiroz» (ESALQ), ubicada en el municipio de Piracicaba, en el Estado de São Paulo. Esa colaboración se tradujo en una suerte de consultoría científica, que se materializó en los debates mantenidos por Toledo Piza Jr. y Octávio Domingues con Renato Kehl sobre los conceptos biológicos manejados en sus publicaciones, así como en la participación de los dos profesores en el Boletim de Eugenia, publicación fundada por Kehl en 1929. La implicación en el periódico llegó a tal extremo, que Toledo Piza Jr. y Domingues asumirían la dirección del boletín en 1930, cuando Kehl emprendió un viaje a Europa.

El análisis de este caso específico tiene como objetivo explorar, en el contexto brasileño, las relaciones establecidas entre la genética y la eugenesia, buscando entender de qué manera en dicho proceso dos profesores de una institución superior de investigación, dedicados al estudio y a la aplicación de los principios de la genética en plantas y animales, vieron en la eugenesia la posibilidad de extender la aplicación de su ciencia en humanos. Por otro lado, es interesante observar cómo un paladín de la eugenesia, Renato Kehl, encontró en Octávio Domingues y Salvador de Toledo Piza Jr. el perfil de investigadores que podría dar mayor fundamento y credibilidad científica a su movimiento.

\section{GENETISTAS Y EUGENISTAS EN BRASIL}

El texto "Eugenics in Brazil, 1917-1940» (Stepan, 1990 $)^{1}$ puede considerarse el hito fundador de los estudios sobre el movimiento eugenésico brasileño, y, después de su publicación, varios libros y artículos comenzaron a abordar la eugenesia en Brasil bajo la perspectiva de la autora norteamericana. De acuerdo con su tesis, la eugenesia latinoamericana, especialmente el movimiento brasileño, tuvo una clara influencia de la eugenesia francesa. La idea de que el individuo, a lo largo de la vida, acumulaba caracteres adquiridos y los transmitía a la generación siguiente predominó entre los eugenistas brasileños. De esta forma, el neolamarckismo permitió un acercamiento a la tradición del sanitarismo y del higienismo. Así, en la segunda década del siglo XX, se entendía la eugenesia nacional en clave optimista. Si los caracteres adquiridos a lo largo de la vida fueran transmitidos a las generaciones futuras, tendríamos, en pocas décadas, una población eugenésicamente sana.

La ecuación 'sanear es eugenizar' fue el leitmotiv del movimiento al final de los años 1910 y la déca- da de 1920. No obstante, Renato Kehl, licenciado en Farmacia y Medicina, y seguramente el defensor más entusiasta de la eugenesia en Brasil, inició al final de la década de 1920 una trayectoria que diverge de esa tendencia general presentada por Stepan. En su estudio «Eugenia negativa e a construção da nacionalidade na trajetória de Renato Kehl (1917-1932)», Vanderlei de Souza pudo advertir que, hacia 1928, Renato Kehl abrazó de manera más clara las teorías mendelianas, en detrimento de argumentos neolamarckistas aún presentes en sus trabajos de principios de la década. A su vez, empezó a negar enfáticamente que sanear fuera eugenizar, ponderando que, si esa confusión había sido útil para una divulgación inicial, aquél era el momento de la afirmación de la autonomía de la eugenesia y del establecimiento de una política que interviniera directamente en las características biológicas de la población. Entre otros elementos de ese cambio, está el viaje de Renato Kehl a Europa, de abril a septiembre de 1928, durante el cual entró en contacto con eugenistas europeos, investigaciones e institutos eugenésicos.

Como director de la Industria Química y Farmacéutica Bayer en Brasil, cargo asumido en 1927, Kehl recibió una invitación para conocer la sede alemana, en el año siguiente. Durante cinco meses el médico recorrió algunos países del norte de Europa y, según Souza, visitó universidades, institutos de antropología y eugenesia. Algunos de ellos fueron el Instituto de Eugenesia de Berlín, en el que Kehl conoció a su director Hermann Muckermann; y el Instituto de Antropología, Genética Humana y Eugenesia de la Universidad Kaiser Wilhelm de Berlín, donde igualmente conoció a Eugen Fisher, director de la institución (Souza, 2006, p. 124). El viaje de Kehl a Europa tuvo importancia capital y fue un elemento decisivo para que el eugenista corroborara las ideas más radicales de la «eugenesia negativa», en especial la concepción pro aria de la eugenesia alemana².

Al año siguiente de su regreso a Brasil, Renato Kehl fundó el Boletim de Eugenia, en consonancia con la nueva concepción de la eugenesia que asumía en aquel momento, diferenciándola del sanitarismo y del higienismo, a la par que proponía medidas más drásticas, como la esterilización de los individuos considerados degenerados. En la misma línea, Kehl publicó el libro Lições de Eugenia, con el objetivo de aprovechar la realización del I Congreso Brasileño de Eugenesia para divulgar su versión más radical de ese polifacético cuerpo de ideas.

Casi diez años más jóvenes que Kehl, Octávio Domingues y Salvador de Toledo Piza Jr. llevaron a cabo su formación académica durante los primeros años de funcionamiento de la Escuela de Agricultura 'Luiz de Queiroz'. Allí se licenciaron y especializaron, res- 
pectivamente en Zootecnia y Zoología, iniciándose ambos en el trabajo de investigación genética con plantas y animales.

Nacido en el $\mathrm{Acre}^{3}$, tras licenciarse por la escuela de Piracicaba en 1917, Octávio Domingues regresó a su región de origen, donde fue contratado como agrónomo por la División de Fomento del Ministerio de Agricultura, e impartió clases en la Escuela de Agronomía de la Amazonía. Siete años después, volvía a la ESALQ y allí se quedaría hasta 1935, cuando sería trasladado a la Escuela Nacional de Agronomía de Río de Janeiro, institución universitaria en la que se jubiló. En su regreso a Piracicaba, en 1924, trabajó como ayudante de Despacho de la 5a cátedra (Zootecnia General, Zootecnia Especial, Exterior y Razas, Bromatología Animal, Lacticinios, Nociones de Higiene y Veterinaria), bajo la dirección del profesor búlgaro Nicolau Athanassof (1878-1955). Al año siguiente, sustituyó a Odilon Ribeiro Nogueira como profesor auxiliar de la asignatura.

Natural de Capivari, municipio del Estado de São Paulo, y también licenciado por la Escuela de Piracicaba, en 1921, Salvador de Toledo Piza Jr. ingresó en el año siguiente en la plantilla de funcionarios, empezando como ayudante de despacho interino de la 5a cátedra (Zootecnia), y pasando en 1931 a profesor catedrático de la 9a cátedra (Zoología General y Especial, Anatomía y Fisiología Comparada de los Animales Domésticos). Como docente e investigador, no conoció otra institución más allá de los campus de la Escuela de Piracicaba. Al año de su graduación, acaecida en 1921, Toledo Piza Jr. viajó a Alemania, donde hizo cursos generales de biología y zoología, y cursos especiales sobre protozoarios e invertebrados en las Escuelas Superiores de Agricultura y Veterinaria de Berlín.

Octávio Domingues y Salvador de Toledo Piza Jr., junto a Nicolau Athanassof, fundaron en 1926 la Revista de Agricultura, publicación periódica que se proponía divulgar "lecciones de agricultura» ${ }^{4}$. Pocos años después, al final de la década de 1920, los dos profesores se incorporaron al movimiento eugenésico. Como buena parte de los genetistas de otros países, los profesores de Piracicaba buscaban presentar la genética como fundamento científico de la eugenesia. Ambos hacían escasas referencias a los principales eugenistas extranjeros, y sus modelos eran, en realidad, genetistas de países que, en algún momento, habían ingresado en el movimiento eugenésico, ofreciendo su trabajo como soporte científico para las medidas propuestas.

Es importante observar que, provenientes de una institución de investigación y enseñanza ubicada en el Estado de São Paulo, Octávio Domingues y Salvador Toledo Piza Jr. forman parte de un contexto específico del movimiento eugenésico en Brasil, que, como han señalado investigaciones recientes, se desarrolló de distintas maneras en diferentes partes del país. En el caso de São Paulo, el pensamiento eugenésico se insertó en una tradición que entendía a los paulistas como resultado de un mestizaje mejor resuelto que en otras regiones. De la mezcla entre blancos e indígenas en el altiplano paulista, ambiente aislado de la costa, habría surgido una población que el movimiento eugenésico del Estado consideraba más sana que la de otras regiones, en las que el negro tenía una presencia mayor (Mota, 2001).

De esa manera, como señalan Maria Gabriela Marinho y André Mota, no es una casualidad que durante el 1 을 Congresso Brasileiro de Eugenia, realizado en 1929, en Río de Janeiro, el psiquiatra paulista Antonio Carlos Pacheco e Silva (1898-1988) señalara en su discurso que "la difusión y propagación de las bases eugenésicas son un esfuerzo predominantemente de São Paulo». Y que "con verdadero orgullo de paulista» (Marinho \& Mota, 2013, p. 204) destacara el hecho de que São Paulo fuera la sede de la primera sociedad eugenésica del país, la Sociedade Eugênica de São Paulo. Dicha Sociedad fue presidida por el médico Arnaldo Vieira de Carvalho y fundada en 1918 gracias al empeño y liderazgo de Renato Kehl -también paulista- personaje central en la discusión desarrollada en este artículo.

El primer registro del contacto entre Renato Kehl y los profesores de Piracicaba es una carta enviada por Octávio Domingues al eugenista con fecha de 22 de julio de 1929, consistente en la respuesta a la carta de Kehl del día 19. En la carta, el zootecnista de Piracicaba lo informaba del envío de $A$ hereditariedade em face da educação, su primer libro sobre eugenesia, solicitado por Kehl, y pedía, a cambio, la obra Lições de Eugenia, también presentado recientemente por el eugenista ${ }^{5}$, durante el referido 1 을 Congresso Brasileiro de Eugenia, que tuvo lugar semanas antes.

Ese fue el inicio de una relación de intercambios científicos e ideológicos basados en la eugenesia y en la genética, teniendo como objetivo principal la «mejora física y moral de la población brasileña» (Stepan, 2005 , p. 170). Conforme las relaciones se iban haciendo más cercanas, Kehl encontraba en los profesores de Piracicaba colaboradores valiosos para la causa eugenésica. Tal vez uno de los mejores ejemplos de ello fuera la invitación a fundar, en 1930, la Comisión Central Brasileña de Eugenesia (CCBE), que se crearía en marzo del siguiente año ${ }^{6}$.

La concepción de la CCBE por parte de Kehl es fruto y reflejo de su contacto y entusiasmo por la eugenesia alemana, que lo llevó incluso a adoptar como modelo la Sociedad Alemana de Raza e Higiene (Stepan, 2005, p. $169)^{7}$. Solamente 10 personas constituían la CCBE, todos elegidos por Kehl ${ }^{8}$. Se observa en este punto la confian- 
za que el eugenista rápidamente pasó a depositar en los genetistas de la ESALQ, situándolos al lado de Belisário Penna y Gustavo Lessa, del Departamento Nacional de Salud Pública, así como de Ernani Lopes, psiquiatra y presidente de la Liga Brasileira de Higyene Mental, con todos los cuales Renato Kehl mantenía vínculos desde tiempo atrás. Según su mentor, la CCBE tendría un amplio abanico de funciones, disponiéndose:

no sólo al estudio y a la propaganda de las cuestiones de carácter eugenésico, sino también a colaborar en cualquier proyecto gubernamental que presente intereses eugenésicos o paraeugenésicos, como los ligados a la inmigración, al poblamiento, a la sanidad, a la educación sexual, a las exigencias modernas prematrimoniales, y a la fundación de establecimientos o laboratorios para estudios galtonianos (Kehl, 1931, p. 1).

No obstante, como veremos, se puede decir que la actuación más efectiva y continua de la CCBE se limitaba a la difusión de la eugenesia por medio del Boletim de Eugenia. En el número de enero de 1930, fue publicado el primer artículo de Octávio Domingues en el Boletim de Eugenia. En su trabajo, Domingues afirmaba que la enseñanza de la genética en las escuelas secundarias, normales y en los cursos superiores, era urgente, una vez que, en palabras suyas, «no es posible hablar de Eugenesia y convencer a un pueblo de las ventajas que alcanzaría la raza con la aplicación de medidas eugenésicas, [...] si dicho pueblo desconoce las bases mismas de esa ciencia y de tales medidas» (Domingues, 1930, p. 2). Domingues creía que la mejor forma de progreso para la eugenesia era la educación, en especial el conocimiento biológico, «cimiento y razón de la eugenesia». La genética aparece, por lo tanto, como punto de apoyo esencial para la comprensión de la importancia de la eugenesia.

Siendo así, Renato Kehl radicalizaba su concepto de eugenesia y se acercaba a Octávio Domingues y Toledo Piza Jr. -profesores que hasta la fecha se habían dedicado a plantas y animales-, quienes creían poder transferir sus conocimientos genéticos a los seres humanos y trasladarlos desde los intramuros de la universidad a la sociedad. Es sin duda un momento estratégico para reflexionar sobre las complejas relaciones entre genética y eugenesia en el contexto específico de Brasil, entre los años 1929 y 1933.

\section{RELACIÓN ENTRE GENETISTAS Y EUGENISTAS: UN CASO EN LOS ESTADOS UNIDOS}

El acercamiento entre eugenesia y genética no fue una característica singular del movimiento eugenésico brasileño. En otros contextos nacionales, genetistas y eugenistas se acercaron en nombre de la ciencia de Galton y de las leyes de Mendel. Los eugenistas buscaron en la naciente genética las bases científicas para sus preceptos. Al considerar al ser humano fundamentalmente como el resultado de su constitución biológica, y al apropiarse de la genética para aplicarla a las sociedades, la eugenesia buscaba ser un puente entre la biología y lo social, así como entre la ciencia pura y la aplicada.

Como observa el historiador Kenneth Ludmerer en su estudio pionero sobre esas relaciones en los Estados Unidos:

de entre estos ejemplos de científicos interesados en las aplicaciones sociales de su disciplina, uno de los casos más destacados es el de los genetistas norteamericanos entre 1905 y 1935. Durante esos años, muchos genetistas se interesaron por la cuestión de si los principios genéticos debían formar parte de las bases de la legislación social, y su preocupación por este asunto los llevó a considerar los logros del movimiento eugenésico (Ludmerer, 1969, p. 337).

No obstante, como veremos, las relaciones entre genética y eugenesia no fueron sencillas. Un caso ejemplar de las aproximaciones y desencuentros entre genetistas y eugenistas se da en la relación entre Charles Davenport (1866-1944) ${ }^{9}$-el mentor del Eugenics Record Office-, y el plant breeder [agronómo] ${ }^{10}$ Willet Hays (1859-1927), en los Estados Unidos del inicio del siglo XX. Ese caso puede servirnos como contrapunto a la colaboración entre Renato Kehl, Octávio Domingues y Salvador de Toledo Piza Jr.

Barbara Kimmelman analizó las relaciones entre eugenesia y genética en los Estados Unidos y el importante papel que la agricultura desempeñó en esa aproximación. La American Breeders Association (1903-1913), dirigida por Willet Hays, fue la primera institución de carácter nacional que relacionó eugenesia y genética en los Estados Unidos. Iniciada por los miembros de la American Association of Agricultural Colleges and Experiment Stations, la ABA tenía como programa de investigación, en un primer momento, los estudios agrícolas y genéticos.

Willet Hays, un heredero del ideario norteamericano de valorización de la tierra y de la agricultura, era simpatizante del Country life movement ${ }^{11}$. De ese modo, conforme escribe Kimmelman, «el mejoramiento agrícola por medio del cultivo era para Hays parte integral de su visión más amplia de la agricultura estadounidense y de la vida rural» (Kimmelman, 1983, p. 170). Hays y su asociación intentaban «el fomento de un modelo idealizado de vida rural para los farmers de América» y, para la realización de ese ideal de la tradición agrarista norteamericana, insistían en «la aplicación de los principios científicos» (Kimmelman, 1983, p. 173-174).

En esa línea, si Hays veía la posibilidad de que la genética fuera aplicada, sería en la mejora de la agricultura y la crianza de animales. De ese modo, «la ABA perseguía extender el enfoque científico a la agricultura entre los practical breeders [cultivadores]» (Ki- 
mmelman, 1983, p. 170). Hays reclamaba que «las increíbles posibilidades de lo que acostumbramos a llamar herencia deben ponerse bajo el control y la dirección del ser humano, dado que constituyen grandes fuerzas físicas de la naturaleza» (Kimmelman, 1983, p. 170). Por lo tanto, de acuerdo con lo que comenta la historiadora, para Hays, «una vez dominadas, tales fuerzas podrían aplicarse al mejoramiento de las especies de plantas y animales, incrementando la calidad y los rendimientos de la producción estadounidense» (Kimmelman, 1983, p. 170).

En 1910, se fundó la American Breeders' Magazine y, a la vez, la eugenesia pasó a formar parte de su programa, en gran medida debido a los esfuerzos del eugenista Charles Davenport (Kimmelman, 1983, p. 184). De acuerdo con Daniel Kevles, Davenport tenía formación en el área de biología, así como en matemáticas, lo que facilitó sus estudios eugenésicos. Antes de dedicarse a los trabajos sobre herencia humana, investigó aves domésticas y canarios. Como indica el historiador, esa investigación:

jugó un papel relevante en los primeros análisis mendelianos de las leyes hereditarias en los animales. Tras trabajar con el color de los ojos, del pelo y de la piel, estaba impaciente por explorar el peso de la herencia en un amplio rango de otras características humanas (Kevles, 1985, p. 45)

De esa forma, el eugenista norteamericano transformó la idea de pedigree, desarrollada a partir de la investigación con animales, en family pedigree, con vistas a sus estudios sobre eugenesia. En 1904, Davenport ya había conseguido financiación para crear un laboratorio de estudios en herencia humana, el Station for Experimental Evolution (SEE), de Cold Spring Harbor, del que fue director. Seis años después, exactamente en 1910, Davenport fundó el Eugenics Record Office (ERO), que se transformaría en un centro de referencia para el movimiento eugenésico norteamericano ${ }^{12}$.

La implicación de Davenport en la ABA ya en la primera década del siglo XX le reportó rápidamente un puesto de influencia allí, favoreciendo la cooperación mutua con Hays y el intercambio entre sus respectivas instituciones (Kimmelman, 1983, p. 184). Con todo, el encuentro entre Hays y Davenport en la ABA partía de motivaciones distintas, porque mientras el segundo se dirigía a la mejora de la sociedad norteamericana por medio del mejoramiento biológico de su población, el geneticista Hays, como hemos visto, veía en la continua expansión de la producción agrícola el camino para el perfeccionamiento del país.

En el caso de Brasil, el estudio del acercamiento entre Kehl y los profesores de la ESALQ, así como el modo en que esa colaboración se concretó en el Boletim de Eugenia, pueden constituir una buena manera de considerar las relaciones establecidas entre genética y eugenesia a finales de la década de 1920 e inicio de la siguiente.

\section{EL BOLETIM DE EUGENIA}

El Boletim de Eugenia tuvo 42 números, publicados entre enero de 1929 y abril-junio de 1933. Renato Kehl era el director y propietario de la publicación periódica, que, inicialmente, fue «editada como propaganda del Instituto Brasileño de Eugenesia». A partir del número 6/7, publicado en junio y julio de 1929, el Boletim de Eugenia pasó a ser una separata de la revista Medicamenta. La dirección y la propiedad siguieron en manos de Renato Kehl, quien lo explicó en una nota, afirmando que uno de los motivos para que el Boletim se convirtiera en un suplemento de la revista era la expansión de los horizontes de la campaña en pro de la eugenesia ${ }^{13}$. A partir de la creación de la CCBE, en marzo de 1931, el Boletim se transformó en su «órgano oficial», y así permaneció desde el número 27 hasta el último, en abril/junio de $1933^{14}$.

Un asunto recurrente en las páginas del Boletim era la herencia y la importancia de su estudio para el mejor conocimiento de los mecanismos de la evolución y la genética, y consecuentemente, en opinión de su director, del perfeccionamiento humano. Las discusiones sobre herencia y genética se realizaban en un lenguaje simple, generalmente para afirmar o rechazar la transmisión de padre a hijo de una determinada enfermedad o patología, pero nunca se practicaba una discusión de mayor calado teórico sobre sus mecanismos. Solía presentar artículos reproducidos de diarios como el Correio da Manhã o el Jornal do Commercio. Renato Kehl dirigió solamente 26 números del Boletim, y hasta su primer contacto con Octávio Domingues por medio de la carta de julio de 1929 , once artículos y notas sobre herencia y genética fueron publicados. Entre esa correspondencia inicial y el comienzo efectivo de la colaboración del profesor de Piracicaba, en enero de 1930, seis artículos y notas fueron publicados sobre el mismo tema, anticipando una nueva fase del Boletim.

Desde sus primeros artículos para el Boletim de Eugenia, Octávio Domingues defendió la existencia de una relación 'visceral' entre agricultura, genética y mejoramiento de plantas y animales, sugiriendo que el éxito de las leyes de Mendel en la agricultura implicaba la posibilidad de mejoramiento en los seres humanos. Para él la enseñanza de la genética permitirá elevar "primeramente el nivel intelectual de nuestras clases cultas en general, y, después, hacer que estas clases fueran más propicias a las ideas que sospechamos resultarían buenas para nuestro mejoramiento eugenésico» (Domingues, 1930, p. 3). Hasta el final de 1930, Domingues publicó más de tres artículos en el Boletim ${ }^{15}$. 
El cambio de contenido en los textos divulgados por la publicación tras el inicio de la colaboración de los profesores de Piracicaba es muy claro. Si antes el objetivo era la divulgación y 'vulgarización' de la eugenesia a partir de textos simples y objetivos, con la paulatina cooperación el Boletim pasó a publicar artículos mayormente dirigidos a las cuestiones científicas tocantes a la genética. Tales trabajos sugerían que la genética había de servir a la eugenesia, ya fuera por medio de la aplicación de las leyes de Mendel al mejoramiento humano, ya por la idea de que la divulgación de la genética contribuiría a romper la resistencia existente en relación con las medidas eugénicas. Junto con ello, el principal cambio fue, desde entonces, dejar de publicar textos afines al neolamarckismo, para privilegiar en sus páginas artículos sobre la genética mendeliana que refutaban la transmisibilidad de caracteres adquiridos.

Poco más de un año después de la publicación del primer artículo de Domingues, en el número de enero de 1930, éste y Toledo Piza Jr. pasarían a ser directores del Boletim. Tras algunos meses de negociaciones acerca del cambio en la dirección del periódico, efectuadas por medio de cartas, en marzo de 1931 los profesores de Piracicaba aceptaron la invitación de Kehl para asumir la dirección del Boletim de Eugenia. Por carta, Domingues informó que el médico seguiría con el puesto de director y que habían optado por publicar el Boletim de Eugenia trimestralmente en formato de revista ${ }^{16}$. Se acordó también que la publicación permaneciera como órgano de propaganda de la CCBE.

A partir del número 37 , publicado en enero-marzo de 1932, bajo la dirección de Octávio Domingues y Salvador de Toledo Piza Jr., el Boletim de Eugenia comenzó a presentar un nuevo formato. Menor en tamaño y mayor en número de páginas, la publicación perdió la característica de un folleto y se acercó al formato de un periódico científico, con artículos más grandes y densos. El objetivo se mantuvo: divulgar la eugenesia. No obstante, la estrategia de hacerlo por medio de la difusión de la genética y de los mecanismos de la herencia salió fortalecida.

La dirección postal del periódico pasó a ser la misma de la Revista de Agricultura, en el municipio de Piracicaba. La portada presentaba los títulos de los artículos publicados, con sus respectivos autores, un modelo común a los periódicos científicos de la época. La contraportada presentaba nuevamente el título, además de la siguiente frase: "Revista trimestral de divulgação e propaganda da eugenia no Brasil. Órgão oficial da Comissão Central Brasileira de Eugenia com sede no Rio de Janeiro».

El primer artículo del número 37 fue un editorial de Renato Kehl presentando la nueva fase del Boletim y alabando a los nuevos colaboradores. Según Kehl, la educación eugenésica había alcanzado a las élites que, hasta entonces, demostraban interés por las leyes de Galton. En su análisis, el escritor paulista escribía:

El Boletim de Eugenia circuló durante tres años entre los intelectuales y estudiosos de casi todos nuestros estados. Consiguió adeptos, muchos de ellos entusiastas, que pasaron a constituir nuevos centros de difusión del ideal regenerador, destacándose, entre ellos, el de Piracicaba, y en su seno sobresalen los profesores Domingues y Piza, dos nuevas celebridades lúcidas y brillantes, que han captado mi atención de eugenista, desde el comienzo mismo de la publicación del Boletim (Kehl, 1932, p. 3).

Con dieciocho páginas, el primer número bajo la dirección de Domingues y Piza presentó aún cuatro artículos más, además de notas, reseñas y noticias relacionadas con el mundo eugenésico. El número 39, publicado en el tercer trimestre de 1932, ya contaba con treinta páginas. Renato Kehl no había dejado de participar efectivamente en el periódico, con su militancia directa en favor de las medidas eugenésicas.

El primer artículo de Toledo Piza Jr. se publicó en el número 38, del trimestre abril-junio de 1932, y representaba claramente la nueva línea del Boletim. Así, ponía el énfasis en la exposición didáctica de la genética, y explicaba el mendelismo, el mecanismo de la herencia y la segregación de los factores genéticos. El autor presentó resumidamente los resultados de los cruces entre una cobaya de pelo rizado y otra de pelo liso, y entre flores blancas y rojas, con las sucesivas generaciones resultantes del cruce. Postulaba exactamente la transposición de experimentos genéticos con animales y plantas a los seres humanos, iniciando una incursión en lo «que puede resultar del casamiento entre el blanco y el negro», título del primero de una serie de artículos que proseguiría en los números siguientes.

Toledo Piza Jr. presentó los resultados de los cruces y de la segregación del gen para el color de la piel a partir de la teoría mendeliana. En ese primer artículo, el autor buscaba explicar, por medio de comparaciones, por qué en la generación resultante del cruce entre un blanco y un negro predominaba el mulato. Más adelante, volveremos sobre los artículos que completarían la serie.

\section{EL PASO DE LA GENÉTICA A LA EUGENESIA}

En septiembre de 1931, en ocasión de su nombramiento como miembro de la American Genetics Association, una entrevista a Octávio Domingues fue publicada en el diario Folha da Manhã y reproducida en el número 33 del Boletim de Eugenia. En ella, el zootecnista hace una defensa de la genética:

Tan grande es su importancia, que sus adeptos defienden que de ese estudio depende el mejoramiento 
del propio hombre, ya que con él se ha conseguido el perfeccionamiento de especies vegetales y animales útiles. Esa pretensión de los eugenistas tiene su fundamento. Es el reflejo o la ilación natural de lo que pasa en el mundo vegetal y en el mundo de los animales domésticos. El mejoramiento de las plantas, con las enseñanzas de la Genética, es una práctica corriente en la agricultura moderna. El perfeccionamiento del ganado, a su vez, es una de las mejores pruebas que se tiene de que los principios de la Genética son verdaderos (Domingues, 1931, p. 3).

A partir de esas consideraciones, Domingues establecía una asociación entre la selección de plantas y animales y el empleo de los conocimientos genéticos en los humanos, para concluir:

Así, trasladar la aplicación de esos conocimientos del mundo animal al hombre, es una medida que se impone por sí misma. Negar que el hombre sea un animal ya no es algo que se permita hoy. Luego, ¿̇por qué no aprovechar esas leyes de la Genética aplicables a los animales, para aplicarlas a los humanos? He aqui por qué los eugenistas esperan mucho de la Genética en el intento de perfeccionar los hijos de Adán en su triple aspecto: físico, intelectual y moral (Domingues, 1931, p. 3).

El pensamiento de Octávio Domingues expresaba cuestiones de extrema relevancia para el período en estudio, como la relevancia atribuida a la genética, considerada la ciencia del siglo XX. La importancia y el éxito de la selección genética en plantas y animales eran el principal argumento para justificar la selección en humanos, uno de los mayores anhelos de Renato Kehl y de los eugenistas en otros países. Aunque los genetistas de varios países se hubieran interesado por saber cómo controlar y entender las leyes de la herencia y crear condiciones para probarlas en humanos, también solían reconocer la dificultad de controlar los rasgos hereditarios en las personas. De acuerdo con ello discurre Daniel Kevles:

En la reproducción sexual, las leyes de probabilidad pueden predecir la frecuencia con que las posibles combinaciones genéticas se dan en los hijos. [...] Las pruebas de hipótesis genéticas han girado en torno a la medición de la frecuencia con que determinados rasgos aparecen en las sucesivas generaciones, aunque la solidez de los test depende de la consecución de un número suficiente de crías de cada generación para determinar todos los posibles resultados genéticos. Así pues, los genetistas de plantas y animales han preferido experimentar con organismos que se reprodujesen prolíficamente ( $y$, de preferencia, con rapidez, con vistas a obtener largas series de generaciones para su análisis) (Kevles, 1985, p. 194).

De ese modo, «la elección de la Drosophila por Thomas Hunt Morgan había otorgado a su programa de investigación genética una ventaja decisiva, dado que las moscas de la fruta satisfacían ambos criterios». Por otro lado, como resume Kevles, «a los genetistas les desagradaba el ser humano como objeto de investigación, puesto que no cumplía con ninguno de ellos» (Kevles, 1985, p. 194).

De hecho, de la misma manera que en otros contextos nacionales, la transposición de experimentos o de resultados de investigación con plantas y animales hacia humanos no era algo tan obvio para los genetistas, como la entrevista de Octávio Domingues daba a entender. Al menos es lo que parece desprenderse de la serie de artículos publicados por Toledo Piza Jr., su colega de la ESALQ.

En el número 39 de la revista, Salvador de Toledo Piza Jr. dedicó seis páginas al estudio iniciado en el artículo anterior, sobre «el casamiento del individuo blanco con el negro a la luz de la biología». El autor explicó en detalle y teóricamente las leyes de Mendel, los cruces sucesivos entre generaciones y las consecuencias provenientes de ellos.

Uno de los objetivos de sus artículos fue probar que el color de la piel en seres humanos no dependía solamente de un único factor mendeliano, sino de la interacción de varios de ellos, no del todo conocidos aún por los genetistas. En palabras suyas, era posible concluir que:

en el cruce blanco x negro ninguno de los colores paternos domina sobre el otro, y además, el carácter 'color de piel' no es el simple producto de la actividad de un único par de genes como en el guisante o en la Mirabilis, sino que depende de un complejo factorial en el que distintas unidades mendelianas entran en juego (Toledo Piza Jr., 1932a, p. 31)

Además de ello, Toledo Piza Jr. tendía a reconocer los límites de la genética, afirmando que «es probable que muchos de los genes del complejo responsable del color de la piel sean dominantes o recesivos para sus alelomorfos, lo que actualmente no puede ser analizado» (Piza Jr., 1932a, p. 31). De esa forma, dado que en aquel momento:

[era] imposible estudiar cada uno de esos factores aisladamente, tendremos que conformarnos con el resultado global, es decir, que de la interacción de los genes de cada padre resulta un individuo cuya piel presenta un color más o menos intermedio. Dicho lo cual, resultará fácil de comprender el motivo por el que sólo raramente reaparecen en la prole de los mestizos los tipos puros correspondientes a los caracteres de las razas de origen, si hacemos una rápida revisión de los fenómenos citológicos de la disociación mendeliana (Toledo Piza Jr., 1932a, p. 31).

Salvador de Toledo Piza Jr. siguió explicando, con figuras ilustrativas sobre el número de pares de cromosomas en las especies, cómo se daba el proceso de herencia en la reproducción, destacando la reducción cromática, y qué ocurría en el cruce de híbridos, para 
comprobar que el color de la piel no se determina por un único par de factores/genes. El autor sostuvo que la observación de descendientes intermedios o híbridos en algunos tipos de plantas y animales, como la oreja de conejos o la semilla de trigo, no significaba verdaderamente la "huida» de las leyes mendelianas de la segregación de los caracteres. Según el autor, esa supuesta excepción a la regla era fruto de una imperfección en el análisis,

[pero] si damos al estudio la profundidad y extensión requeridas, llegaremos a descubrir la causa de la aparente indisociabilidad de los caracteres del híbrido,

los cuales, en realidad, también están sujetos a las mismas leyes que rigen los fenómenos hereditarios en general (Toledo Piza Jr., 1932b, p. 62).

Con una tabla en la que presentó los posibles resultados -sesenta y cuatro posibilidades-del cruce entre conejos de orejas de distintos tamaños, Toledo Piza Jr. demostró que esas combinaciones no escapaban a las reglas de las leyes de Mendel, sino que formaban parte de procesos más complejos de herencia mendeliana. El mismo razonamiento sería válido para el color de la piel en los seres humanos.

En el número 41 de la revista, publicado en el primer trimestre de 1933, el estudio se concluyó en ocho páginas más, discutiendo, finalmente, el casamiento entre individuos blancos y negros. En palabras de Toledo Piza Jr., el color de la piel fue elegido como ejemplo para el estudio, ya que sería el carácter más «palpable» y, añadía, el más "chocante». Aun de acuerdo con el autor, el estudio de la genética humana, en aquel momento, quedaría restringido a la simple observación y a la transposición de los experimentos con plantas y animales a la realidad de los hombres. Como afirmaba, "no queda la más mínima duda de que, siendo el hombre un animal, como tal se comporta, y reacciona al medio de manera fundamentalmente idéntica a los demás animales» (Toledo Piza Jr., 1933a, p. 6). Sin embargo, diferentemente de Octávio Domingues, Toledo Piza Jr. destacaba que:

Pretender, no obstante, que bajo cierto y determinado punto de vista, el hombre, sólo por ser animal, se comporte como otro animal cualquiera, como un gusano o un insecto, sin considerar las enormes diferencias que entre ellos existen, reflejadas en la diversidad de los rumbos que cada uno siguió bajo el influjo de los factores que rigen la evolución de todos los seres, es lo que definitivamente no podemos admitir. De ahí el peligro de las generalizaciones (Toledo Piza Jr., 1933a, p. 6).

Así, por un lado, afirmaba que "en materia de genética [...] no se puede experimentar con el hombre», y, por otro lado, seguía defendiendo que «la observación de muchos casos bien controlados nos indica que, bajo el punto de vista hereditario, el hombre está sujeto a las mismas leyes básicas generales que cabe aplicar a los demás animales y plantas» (Toledo Piza
Jr., 1933a, p. 6). De este modo, a pesar de todos los límites del conocimiento de la genética humana,

[al menos ya se había] constatado que un gran número de caracteres humanos se transmiten de padres a hijos según los preceptos mendelianos. En el hombre, como en los animales y en las plantas, hay genes dominantes y genes recesivos; en él, como en las plantas y en los animales, el mismo mecanismo citológico separa los cromosomas homólogos en el decurso de la gametogénesis, distribuyendo por células reproductoras distintas los factores de un mismo par; en el hombre también hay factores asociados (Toledo Piza Jr., 1933a, p. 6)

De ese modo, al mismo tiempo que anunciaba un camino prometedor, Toledo Piza Jr. concluía:

Sin embargo, a pesar de todo, no sin riesgo de equivocarnos, extendemos sobre el hombre conclusiones de experimentos realizados con moscas creadas dentro de frascos en las condiciones artificiales de los laboratorios. Cuando todo parece indicar que él se ajusta perfectamente a los moldes de una teoría forjada en un frasco de cultivo, pudiendo su comportamiento genético ser comprendido y explicado de pleno acuerdo con esa teoría, nadie podrá garantizar que en realidad sea realmente así (Toledo Piza Jr., 1933a, pp. 6-7).

Siguiendo su presupuesto acerca de la imposibilidad de realizar experimentos genéticos con seres humanos, y sin embargo, estando suficientemente comprobado que las leyes mendelianas eran válidas para los humanos, Toledo Piza Jr. realizaba el ejercicio de transferencia de resultados de experimentos con animales a los hombres. En el caso que le interesaba, tomó como ejemplo el albinismo en moscas y los tipos que descienden del cruce entre moscas albinas y no albinas, y, a partir de ello, discutió el comportamiento genético del color de la piel en la descendencia del mulato.

En las últimas páginas de su artículo, revela por qué, a pesar de la claridad científica de las leyes mendelianas de la herencia y de su validez para los humanos, era necesario tener prudencia al tratar con ellos. En el caso en cuestión, observa que «también sobre la pigmentación influye el aparato endocrino» (Toledo Piza Jr., 1933a, p. 10). Aunque la actuación de las glándulas "en nada contraría» las observaciones establecidas a partir de las leyes de Mendel,

por el injerto de glándulas o por la extirpación, por la inyección de extractos o administración per os [sic] de fragmentos glandulares, se ha modificado la colocación del tegumento o de sus producciones, en la dirección del albinismo o del melanismo (Toledo Piza Jr., 1933a, p. 10).

Más que la alteración de los resultados de las combinaciones mendelianas, sin llegar a negarlas, lo que aparentemente permite a Toledo Piza Jr. desviarse en su estudio de las conclusiones sugeridas por los análisis de los factores mendelianos, es la introducción 
de la acción del "aparato endocrino», dado que él considera que «los productos elaborados por las distintas glándulas de secreción interna influyen poderosamente sobre el [...] metabolismo general» (Toledo Piza Jr., 1933a, p. 10).

En ese camino, la consecuencia del cruce de blancos y negros resultaba en un mayor porcentual de mulatos, tal como había sido sugerido por los experimentos con el tamaño de las orejas de conejos o con las semillas de trigo. En todos esos casos, la generación de descendientes con características intermedias era perfectamente explicable por la combinación de factores genéticos, y llevaba a la constatación de la normalidad de esos híbridos. No obstante, tras exponer los comentarios sobre la acción de las glándulas y reafirmar la complejidad del ser humano, considera, que «el blanco y el negro son tan distintos bajo tan variados aspectos, que bien podrían ser considerados pertenecientes a especies distintas» (Toledo Piza Jr., 1933a, p. 10)

Toledo Piza Jr. buscaba amparar esa concepción considerando que, "en verdad, para un gran número de animales, hemos sido mucho menos rigurosos al incluir en especies distintas seres mucho más afines» (Toledo Piza Jr., 1933a, p. 10). Y a partir de la concepción de que blancos y negros pertenecen a especies distintas, concluye que:

el casamiento del individuo blanco con el negro y de los mulatos entre sí, es como un cruce interespecífico, es decir, un verdadero polihibridismo complicado por un elevado grado de heterocigosis de un gran número de factores de cada una de las fuentes iniciales (Toledo Piza Jr., 1933a, p. 10)

De acuerdo con su observación de que los seres humanos blancos y negros no pertenecían a la misma especie, deducía que, bajo el «punto de vista antropológico», "las uniones de blancos con negros no son naturales». Además de eso, el autor era categórico al afirmar que, bajo el «prisma social», «el casamiento del individuo blanco con el negro, cualesquiera que fueran las ventajas que de él obtuviera el individuo o la comunidad, debería considerarse, en la situación presente, una unión repugnante» (Toledo Piza Jr., 1933a, p. 12). Esa era la conclusión de su serie de artículos.

Por otro lado, partiendo de la misma teoría y de la misma ciencia -la genética mendeliana-, Domingues desarrolló una visión distinta del mestizaje racial. Mientras Toledo Piza Jr. afirmaba categóricamente que el mestizaje era perjudicial a la raza, que los casamientos entre individuos blancos y negros, eran 'repugnantes' bajo el punto de vista social, para Domingues, la mezcla no suponía ningún perjuicio. Domingues lo explicó a partir de la demostración de cómo serían los genotipos de las generaciones F1 y F2, en el cruce entre blancos, negros y mulatos. La conclu- sión del zootecnista fue que el mestizaje contribuiría al blanqueamiento de la población brasileña, como preveían y deseaban algunos especialistas:

He aquí explicada la desaparición del negro en Brasil, pronosticada para dentro de algunos años, mientras que en América del Norte la evolución del negro africano, en un ambiente avanzado, constituye un problema inquietante para los americanos (Domingues, 1929, p. 91).

Octávio Domingues fue más allá. Contrariando el precepto compartido por algunos eugenistas, incluso por su colega de institución, -el de que existirían razas superiores e inferiores- aún en 1929 escribió:

Existe un prejuicio muy tonto, y muy vulgarizado, que confiere a las razas puras (pseudo-puras, se diría mejor) una superioridad absoluta sobre los núcleos populosos, formados por mestizaje actual. No sé de dónde proviene idea tan sin fundamento científico.

[...] Bueno, entre humanos, esa pureza de la raza no tiene importancia para el mejoramiento de la especie, ya que todas las razas humanas están, más o menos, manchadas por taras, malformaciones, herencias psicológicamente malas. Luego, no podemos ligar la idea de superioridad a la idea de raza pura (Domingues, 1929, p. 136).

En ese sentido, es posible comprender mejor su defensa de la educación eugenésica, contraria a las propuestas de implementación de medidas eugenésicas radicales. Nancy Stepan Ilamó la atención sobre esa diferencia del pensamiento de Domingues en relación con Renato Kehl (podríamos añadir que también con relación a Toledo Piza Jr.), y afirmó que el zootecnista formaba parte de una línea de pensamiento que puede entenderse como «una interesante anticipación de la tesis de Gilberto Freyre sobre la 'democracia racial' en Brasil, con su dependencia de la biología racial y su visión positiva del mestizaje, entendiéndolo como una forma de eugenización» (Stepan, 2005, p. 171). Del lado de Octávio Domingues se alineaba el antropólogo Edgar Roquette-Pinto, que en 1929, también amparado en el mendelismo, afirmó en el I Congreso Brasileño de Eugenesia que los cruces entre blancos y negros eran «normales y sanos» (Stepan, 2005, p. 171).

Con artículos de carácter incisivo y propagandístico, Octávio Domingues era más directo al establecer la correlación entre genética y eugenesia, trasladando lo que se sabía sobre plantas y animales a los humanos. Por otro lado, consideraba que las leyes mendelianas de segregación de los genes y su posterior recombinación en pares -y no mezcla- hacía que todo híbrido -sea planta o animal- fuera normal; incluido el mulato, en el caso del ser humano. A su vez, Salvador de Toledo Piza Jr. adoptaba un estilo de escritura más cercano a un artículo científico y se presentaba más como genetista. En esa línea, reforzaba la complejidad 
del funcionamiento de las leyes mendelianas en los humanos, así como la acción de las glándulas, lo que exigiría ciertos cuidados en el camino que llevaba de la genética a la eugenesia. No obstante, partiendo de la misma teoría científica que su colega, consideraba al mulato como una aberración, toda vez que, en su visión, era el resultado de la combinación de especies distintas ${ }^{17}$

\section{ALEJAMIENTOS}

A pesar de las diferencias entre las concepciones de Domingues y Toledo Piza Jr., cabe afirmar que el Boletim de Eugenia fue un espacio en el que la eugenesia, por medio de Renato Kehl, y la genética, con Domingues y Toledo Piza Jr., convivieron de modo articulado y con escasas tensiones.

En el último número publicado, en el trimestre de abril/junio de 1933, Salvador de Toledo Piza Jr. volvía a destacar la diferencia entre eugenesia y genética. Para él, "la eugenesia no es la genética humana ya que le falta sobre todo la parte experimental» (Toledo Piza Jr., 1933b, p. 16). Por otro lado, la eugenesia era «la ciencia que tiene por objetivo aplicar al hombre los principios útiles que la genética recoge de su continua experimentación con los animales» (Toledo Piza Jr., 1933b, p. 16). Y proseguía:

Así concebida, la eugenesia es una ciencia aplicada; es una verdadera zootecnia humana. La zootecnia tiene por objeto mejorar económicamente los animales domésticos, creándolos a la luz de las enseñanzas promanadas de la genética; la eugenesia pretende el mejoramiento social del hombre, conduciéndolo bajo

la misma luz (Toledo Piza Jr., 1933b, p. 16).

Como vimos, en los Estados Unidos del inicio del siglo XX, el acercamiento entre Hays y Davenport se dio con el reconocimiento de las diferencias entre la genética y la eugenesia, además de la inexistencia de una afinidad absoluta en las intenciones de los actores implicados. Se puede decir que en la ABA no hubo una confluencia integral entre la eugenesia y la genética. El ocaso de la ABA tuvo lugar entre 1910 y 1913 por una serie de factores, incluido el desinterés de Davenport por participar en la Asociación. El eugenista norteamericano ya había conseguido el apoyo necesario para el desarrollo de sus estudios eugenésicos, además de alimentar divergencias con Hays sobre los rumbos de la Asociación, que fue reorganizada en 1913 y pasó a llamarse American Genetics Association, la misma institución a la que Octávio Domingues se dirigió en 1931.

De hecho, los ideales de Hays y de Davenport nunca confluyeron de manera integral, ya que el genetista defendía la aplicación de las leyes de Mendel en el mejoramiento de las plantas y de los animales, proporcionando así una renovación moderna y científica a la tradición del agrarismo norteamericano; mientras que el eugenista defendía la posibilidad de aplicar los conocimientos genéticos en el mejoramiento biológico del hombre, en consonancia con el ideal del nuevo hombre eugenizado (Kimmelman, 1983, p. 184).

Por fin, alejado de la ABA y de Hays, Charles Davenport se dedicó durante tres décadas al movimiento eugenésico y al ERO. Durante ese período recolectó miles de datos sobre la población, analizando pedigree, herencia, raza y otros asuntos afines a los eugenistas en las primeras décadas del siglo XX.

En Brasil, el ideal compartido por Toledo Piza Jr., Octávio Domingues y Renato Kehl era dar efectividad a la CCBE, de acuerdo con los moldes del ERO, esa organización promovida por Davenport al disociarse de Hays y de su institución de genética aplicada a plantas y animales. En el caso que nos concierne, los profesores de la ESALQ estarían del lado de Kehl en ese tipo de empresa. Incluso, como hemos destacado, el mismo Boletim de Eugenia era considerado un órgano difusor de la CCBE desde que ésta fuera creada, y así permaneció después de que Domingues y Toledo Piza Jr. asumieran sus funciones en la dirección de la redacción del periódico.

De todos modos, si afirmamos que la genética tuvo una asociación menos tensa con la eugenesia, representada en las figuras de Kehl, Domingues y Toledo Piza Jr., en comparación con la disociación entre Hays y Davenport, es necesario reconocer que el propio Toledo Piza Jr. consideraba ese éxito muy limitado. Entre los brasileños, aunque hayan publicado cartillas, la acción ligada a la propaganda prácticamente se resumió al Boletim de Eugenia ${ }^{18}$. En cuanto al objetivo de colaborar con proyectos gubernamentales, el mayor triunfo obtenido por la CCBE probablemente fuera la invitación realizada a Kehl para que, en 1932, integrara el grupo liderado por Oliveira Vianna (1883-1951), que elaboró el proyecto de inmigración y poblamiento, aprobado por el Congreso Nacional en 1935. En relación con el plan de fundación de establecimientos o laboratorios, Kehl estaba planeando, por lo menos desde 1929, la creación de un Instituto Brasileño de Eugenesia, inspirado en el Instituto de Eugenesia de Berlín (Kehl, 1929, p.1), el cual nunca llegó a hacerse efectivo.

Para Toledo Piza Jr., los límites de la eugenesia en Brasil parecían ser los del propio Boletim. De acuerdo con sus consideraciones, el periódico sólo consiguió cosechar algunos pocos entusiastas de la ciencia de Galton, que muchas veces eran mal interpretados en sus propuestas de medidas eugenésicas. En su visión, el desconocimiento de las leyes de la genética y de las intenciones de la eugenesia, conducía a la debilidad del movimiento y lo llevaba a constatar, con cierto desánimo, que «mientras la zootecnia progresa a pa- 
sos agigantados, alcanzando resultados sorprendentes, la eugenesia a partir de Galton sólo ha conseguido resultados literarios, sin ningún progreso palpable en el campo de la utilidad» (Toledo Piza Jr., 1933b, p. 16).

Pero quizás el desánimo de Toledo Piza Jr. con Brasil pudiera ser compartido por los eugenistas de otros países. Aunque la instauración de la ley alemana de esterilización de degenerados fuera instituida en el año de 1933, y recibida con entusiasmo por eugenistas de todo el mundo, los vientos no eran tan favorables a la eugenesia. En el caso de los Estados Unidos, por lo menos en la lectura del historiador Garland Allen, en esa década en que ya se anunciaba la Segunda Guerra Mundial, Davenport afrontaba críticas a los procedimientos de investigación del ERO, ausencia de pruebas científicas sobre la herencia humana, además de cuestiones económicas y falta de financiación, lo que provocó que su institución entrara en decadencia (cf. Allen, 1986, pp. 250-254).

Exactamente en ese período, «los genetistas académicos comenzaron a incidir en la polémica acerca de las diferencias genéticas entre razas y grupos étnicos, surgida como consecuencia de los debates en torno a la inmigración» (Allen, 1986, p. 250), una convicción que, como hemos visto, permanecía en Salvador de Toledo Piza Jr., pero no en Octávio Domingues.

Sin embargo, oponiéndose a los estudios de Garland Allen, la investigadora Diane Paul argumenta que la creencia de que rasgos de personalidad, inteligencia y modelos de comportamiento venían genéticamente determinados se mantuvo durante algún tiempo entre los genetistas. Así, aún en el transcurso de la década de 1920 y 1930, faltaba apenas un paso para seguir acercándose a los ideales eugenésicos.

Hay que destacar que Diane Paul disiente de Garland Allen a partir de su estudio sobre el norteamericano Hermann J. Mueller (1890-1967), autor citado por Allen como uno de los genetistas que pronto se alejaron de la eugenesia. Por otro lado, Paul argumenta que "fue el científico más activamente implicado en el desarrollo de una eugenesia socialista durante las décadas de 1920 y 1930» (Paul, 1984, p. 575). También es importante observar que esta corriente consideraba que, "sin una revolución económica y social, no habrá una revolución en nuestras actitudes hacia el sexo y la reproducción» (Paul, 1984, p. 578), lo que no deja de reflejar el desánimo de eugenistas de distintos países frente a políticas que consideraban demasiado liberales, o incluso a Estados con políticas de protección social.

En rigor, no era distinta la suerte del movimiento eugenésico más radical en Brasil. En su evaluación de la historia de la eugenesia, Gilberto Hochman, Nísia Trindade Lima y Marcos Chor Maio destacan que a partir de 1930, en el período de la presidencia de Getúlio Vargas (1882-1954), ocurrieron varios cambios institucionales que establecieron un nuevo panorama jurídico y un sistema de protección social: la nueva legislación laboral, la reglamentación de la jornada de trabajo, la seguridad social, las vacaciones, la jubilación para los trabajadores urbanos, un sueldo mínimo interprofesional, y la asistencia a las madres, los niños y la familia (Hochman, Lima \& Chor Maio, 2010, p. 504). Como comentan los autores, por un lado,

Algunas de las demandas más antiguas de los eugenistas en pos de un mayor activismo del Estado pueden encontrarse a lo largo de varios proyectos de Vargas, sobre todo a través de las acciones relacionadas con la protección maternal y de la infancia, que resultaban clave para un gobierno que articuló el papel especial de la infancia, una idea general de raza, así como de construcción de la nación (Hochman, Lima \& Chor Maio, 2010, p. 504).

\section{Mientras que por otro,}

La Asamblea Nacional que aprobó la nueva Constitución Brasileña de 1934 debatió las propuestas de la eugenesia negativa para legalizar el aborto en casos excepcionales y el control de la natalidad por razones eugenésicas, si bien no fueron aprobadas (Hochman, Lima \& Chor Maio, 2010, p. 504).

En ese contexto, hay que señalar que el Boletim de Eugenia dejó de publicarse en 1933. El número en el que apareció el artículo de Toledo Piza Jr., y que refería los límites del éxito de la asociación entre genetistas y eugenistas, fue el último de la publicación.

\section{CONSIDERACIONES FINALES}

Nuestro objetivo ha sido reflexionar sobre la relación entre genética y eugenesia en Brasil a partir de un caso específico, el de la relación entre un zootecnista, un zoólogo y un médico. El Boletim de Eugenia, una publicación periódica que se proponía divulgar y popularizar la eugenesia en Brasil, fue el punto de encuentro entre Renato Kehl y los genetistas de la ESALQ. Poco a poco, sufrió cambios con la incorporación de Octávio Domingues y Salvador de Toledo Piza Jr. al cuerpo de colaboradores y, sobre todo, como protagonistas activos de la campaña eugenésica. Uno de los cambios más perceptibles fue el énfasis dado a las discusiones sobre genética mendeliana. Los artículos sobre el tema se volvieron más teóricos, con la presentación de resultados de investigaciones con animales y plantas, muchas veces acompañados de gráficos y tablas demostrativos.

Además de la creencia en la eugenesia y en la ciencia, para los profesores de Piracicaba, el movimiento eugenésico pudo verse como una forma más de difusión de la ciencia, de la ESALQ y de sus nombres. A su vez, el conocimiento y la familiaridad de ambos 
con las cuestiones de genética animal y vegetal y con el debate sobre la herencia humana debió de haber pesado en la decisión de Kehl de acercarse a los genetistas, al punto de invitarlos a que participaran en el grupo que constituyó la CCBE, así como a que integraran la dirección del Boletim de Eugenia.

El caso de Octávio Domingues y Salvador de Toledo Piza Jr., que se acercaron a la eugenesia reconociéndose y siendo reconocidos como genetistas, puede recordarnos, en alguna medida, el caso de Willet Hays. Aunque su acercamiento a la eugenesia ocurriera en un período anterior, la comparación es válida por el hecho de que el plant breeder norteamericano tenía un perfil semejante al de nuestros dos autores. Sin embargo, esto no debe significar una generalización, ya que no estamos comparando dos casos nacionales de relaciones establecidas entre genetistas y eugenistas.

En el caso específico de Hays, el genetista norteamericano no llegó a ser totalmente afín al movimiento eugenésico, debido, en gran medida, a sus creencias en torno al ideal agrícola, tan fuerte en el imaginario norteamericano; mientras que los brasi-

\section{NOTAS}

1 En 1991, Nancy Stepan publicó el libro The hour of eugenics: race, gender and nation in Latin América (Stepan, 1991). Tanto el texto de 1990, como el libro de 1991, fueron traducidos al portugués. Una primera versión del texto de la autora fue publicada en la Revista Quipu, en 1985. Es importante destacar que el trabajo de Stepan integraba un proyecto amplio de investigación sobre la eugenesia en distintos contextos nacionales, coordinado por Mark Adams y que resultó en el libro The Wellborn Science (Adams, 1990).

2 Además de realizar un estudio detallado sobre la trayectoria intelectual del final de los años 1920 y sobre la importancia del viaje a Europa para Renato Kehl, Souza discute la importancia del determinismo biológico y de la herencia como base de la eugenesia en ese período (Souza, 2006).

3 Actual Estado de la región Norte, en la Federación Brasileña. N.T.

4 La expresión 'lecciones de agricultura' ha sido entresacada del texto editorial de la Revista de Agricultura, 1926, p. 1 (Habib, 2010).

5 Correspondencia de Octavio Domingues a Renato Kehl. Piracicaba, 22/07/1929 (Departamento de Arquivo e Documentação/Casa de Oswaldo Cruz/FIOCRUZ). Domingues publicó leños, en la creencia de estar imbuidos solamente del ideal científico, abrazaron con más entusiasmo la eugenesia. Como hemos visto, especialmente en el caso de Salvador de Toledo Piza Jr., ese acercamiento era defendido aun cuando reconocía las limitaciones de los resultados de las investigaciones genéticas con humanos.

Por otro lado, aunque la vinculación entre genética y eugenesia fuera mayor, sus resultados quedaron restringidos a la publicación de un periódico de difusión del movimiento, y Renato Kehl no consiguió dar vida a la CCBE como le habría gustado, al contrario que su colega norteamericano Charles Davenport que, sin el apoyo de Willet Hays, fundó y dirigió su propio centro de investigaciones, el ERO. De todas formas, el grito de Salvador de Toledo Piza Jr. en el último número del Boletim de Eugenia parece haber sido el canto del cisne del movimiento eugenésico más radical de Brasil.

\section{AGRADECIMIENTOS}

Artículo traducido al castellano por Bethania Guerra de Lemos y Juan Bautista Rodríguez Aguilar.

tres libros sobre eugenesia (Domingues, 1929, 1933, 1936), (Stefano, 2009).

6 Tanto Octavio Domingues como Toledo Piza Jr. declaran, en cartas a Renato Kehl, que aceptan formar parte de la CCBE, afirmando estar de acuerdo con los Estatutos enviados por el eugenista. Ver: Correspondencia de Octavio Domingues a Renato Kehl. Piracicaba, 01/03/1931; Correspondencia de Salvador de Toledo Piza Jr. a Renato Kehl. Piracicaba, 06/03/1931. Todas las correspondencias: Departamento de Arquivo e Documentação/ Casa de Oswaldo Cruz/FIOCRUZ.

7 Más tarde, en un artículo de 1956, Kehl afirmaría que la «Comisión Central Brasileña de Eugenesia seguiría el modelo del Instituto Americano de Eugenesia, dirigido por C. Davenport» (Kehl, 1956).

8 «Noticiário - Comissão Central Brasileira para o Estudo e Propaganda da Eugenia», Archivos Brasileiros de Hygiene Mental, 4 (2), marzo-abril-mayo de 1931, p. 153

9 Charles Benedict Davenport, principal eugenista norteamericano en las primeras décadas del siglo XX, se licenció en ingeniería en 1887 y luego obtuvo, en 1892, su Doctorado en Biología por la Universidad de Harvard. Habiendo trabajado en el laborato- 
rio de Karl Pearson (1857-1936), en Londres, fundó en 1910 el Eugenic Record Office (ERO), en Cold Spring Harbor. Para más informaciones ver Allen (1986) y Cruz (2013). El eugenista y sus estudios ejercieron una gran influencia en el movimiento eugenésico brasileño, inspirando no solo a médicos y genetistas como Renato Kehl, Octavio Domingues y Salvador de Toledo Piza Jr., sino también a antropólogos como Edgar Roquette-Pinto (Souza, 2011).

10 Sobre el término plant breeder, no hay una expresión que abarque su significado. Es importante observar que en una pequeña rectificación en el Boletim de Eugenia, el cargo de Profesor de Zootecnia ocupado por Octavio Domingues fue traducido al inglés como 'Professor of Animal Breeding' ("Rectification», Boletim de Eugenia, 3 (32), agosto de 1931, p. 7). Desde este punto de vista, se podría traducir plant breeder por agrónomo.

11 En las primeras décadas del siglo XX, el Country life movement reunió científicos, periodistas, políticos y agricultores alrededor de la búsqueda del mejoramiento de las condiciones de vida y de producción en las regiones rurales de los Estados Unidos.

12 Es importante observar cómo Daniel Kevles presentó los intereses personales y los esfuerzos de Davenport por establecer contactos profesionales durante su 'batalla' por el desarrollo de la eugenesia, con la creación de un laboratorio y por la financiación de ricas e importantes figuras de la sociedad norteamericana, además del interés en mantener el contacto con colegas de otros países (Kevles, 1985, p. 45).

13 "Boletim de Eugenia e 'Medicamenta'», Boletim de Eugenia, 1 (6/7), junho-julho de 1929, p. 1
14 Deberían ser suscriptores del periódico «quienes así lo desearan», como decía el anuncio. La suscripción anual del ejemplar suelto del Boletín costaba cinco mil réis [el nombre de la moneda oficial de Brasil en la época. N.T.], valor relativamente bajo si se lo compara a las suscripciones de otras revistas del mismo período. Como refería Renato Kehl en una carta de 1932, dirigida a Octavio Domingues, el Boletim se remitía a los suscriptores, y también era enviado "sistemáticamente a las instituciones eugenésicas extranjeras y a los partidarios nacionales de la eugenesia, reservando los ejemplares sobrantes a la distribución entre intelectuales, miembros del gobierno, periódicos, escuelas, etc.» (Correspondencia de Renato Kehl a Octavio Domingues. Río de Janeiro, 10/03/1932. DAD/COC/FIOCRUZ).

15 Mientras tanto, la primera colaboración de Salvador de Toledo Piza Jr. fue la publicación de un pequeño resumen de su Teoría de los Plastinemas, en francés, con el objetivo de facilitar la divulgación de la investigación en el exterior (Toledo Piza Jr., 1930, pp. 4-5).

16 Correspondencia de Octavio Domingues a Renato Kehl. Piracicaba, 05/03/1932. DAD/COC/FIOCRUZ.

17 Aunque admitiera que «de hecho, tanto el blanco como el negro vienen, hace milenios, reproduciéndose en una verdadera promiscuidad de razas» consideraba que seguían formando especies distintas, generando su mezcla «los resultados más imprevistos e inexplicables» (Toledo Piza Jr., 1933a, p. 10-11).

18 En 1932 se publicó la primera (y probablemente la única) cartilla organizada por la CCBE. De autoría de Renato Kehl y titulada Educação Eugênica, se publicó en Río de Janeiro por la editorial Livraria Francisco Alves.

\section{BIBLIOGRAFÍA}

Adams, Mark (ed) (1990), The Wellborn Science. Eugenics in Germany, France, Brazil and Russia, New York, Oxford University Press.

Allen, Garland (1986), "The Eugenics Record Office at Cold Spring Harbor, 1910-1940. An Essay in Institutional History", Osiris, $2^{\text {nd }}$ Series, 2, pp. 225-264.

Cruz, Rodrigo Andrade da (2013), "Das ervilhas mendelianas ao 'décimo submerso'. Aspectos teóricos e práticos do desenvolvimento da eugenia nos Estados Unidos". En: Mota, André; Marinho, Maria Gabriela S.M.C. (eds.), Eugenia e História: ciência, educação e regionalidades, São Paulo, Faculdade de Medicina da USP, Universidade Federal do ABC, CD.G Casa de Soluções e Editora, pp. 37-48.

Domingues, Octávio (1929), Hereditariedade e Educação, São Paulo, Melhoramentos.

Domingues, Octávio (1930), "Os Programas de Ensino e a Genética”, Boletim de Eugenia, 2 (13), pp. 2-3.

Domingues, Octávio (1931), "Em torno dos problemas eugênicos". Boletim de Eugenia, 3 (33), p. 3.
Domingues, Octávio (1933), Eugenia: seus propósitos, suas bases, seus meios, São Paulo, Companhia Editora Nacional.

Domingues, Octávio (1936), Hereditariedade e Eugenia, Rio de Janeiro, Civilização Brasileira.

Habib, Paula Arantes Botelho Briglia (2010), Agricultura e Biologia na Esvola Superior de Agricultura 'Luiz de Queiroz' (ESALQ): os estudos de genética nas trajetórias de Carlos Teixeira Mendes, Octávio Domingues e Salvador de Toledo Piza Jr. (1917-1937), Rio de Janeiro, Tese de Doutorado em História das Ciências e da Saúde, Casa de Oswaldo Cruz/Fiocruz.

Hochman, Gilberto; Lima, Nísia Trindade; Chor Maio, Marcos (2010), "The Path of Eugenics in Brazil: dilemmas of miscegenation". En: Bashford, Alison; Levine, Philippa (eds.), The Oxford Handbook of the history of eugenics. Oxford, Oxford University Press, pp. 493-510.

Kehl, Renato (1929), "O Instituto Brasileiro de Eugenia”, Boletim de Eugenia, 1 (2), p 1. 
Kehl, Renato (1931), "Uma nova entidade cientifica que aparece: A comissão central brasileira de eugenia", Boletim de Eugenia, 3 (27), p. 1.

Kehl, Renato (1932), "A Nova Fase do Boletim de Eugenia", Boletim de Eugenia, 4 (37), p. 3.

Kehl, Renato (1956), "A sementeira humana", A Gazeta, São Paulo, 16 de maio.

Kevles, Daniel (1985), In the Name of Eugenics. Genetics and the Uses of Human Heredity, Berkeley and Los Angeles, University of California Press.

Kimmelman, Barbara (1983), "The American Breeders' Association: Genetics and Eugenics in an Agricultural Context, 1903-1913", Social Studies of Science, 13 (2), pp. 163-204.

Ludmerer, Kenneth (1969), "American Geneticists and the Eugenics Movement: 1905-1935", Journal of the History of Biology, 2 (2), pp. 337-362.

Marinho, Maria Gabriela S.M.C.; Mota, André (2013), "Tramas e teias da retórica eugênica em São Paulo. Personagens, Contextos e Instituições Médicas (1916-1954)". En: (eds.), Eugenia e História: ciência, educação e regionalidades, São Paulo, Faculdade de Medicina da USP, Universidade Federal do ABC, CD.G Casa de Soluções e Editora, pp. 201-218.

Mota, André (2001), Tropeços da Medicina Bandeirante: São Paulo, 1892-1920, São Paulo, Tese de Doutorado em História, Universidade de São Paulo.

Paul, Diane (1984), "Eugenic and the Left", Journal of the History of Ideas, 45 (4), pp. 567-590.

Reis, José Roberto Franco (1994), Higiene mental e eugenia: o projeto de "regeneração nacional" da Liga Brasileira de Higiene Mental (1920-30), Campinas, São Paulo, Dissertação de Mestrado, Programa de Pós-Graduação em História, Universidade Estadual de Campinas.
Souza, Vanderlei de (2006), A Política Biológica como Projeto: a 'eugenia negativa' e a construção da nacionalidade na trajetória de Renato Kehl (1917-1932), Rio de Janeiro, Dissertação de Mestrado em História das Ciências e da Saúde, Casa de Oswaldo Cruz/Fiocruz.

Souza, Vanderlei de (2011), Em Busca do Brasil: Edgard RoquettePinto e o retrato antropológico brasileiro (1905-1935), Rio de Janeiro, Tese de Doutorado em História das Ciências e da Saúde, Casa de Oswaldo Cruz/Fiocruz.

Stefano, Waldir (2009), "Octávio Domingues: concepções sobre miscigenação no contexto eugenésico". Temas e Matizes, 15, pp. 42-54.

Stepan, Nancy (1990), "Eugenics in Brazil, 1917-1940". En: Adams, Mark B. (ed.), The Wellborn Science: eugenics in Germany, France, Brazil and Russia, New York, Oxford University Press.

Stepan, Nancy (2005), A hora da Eugenia: raça, gênero e nação na América Latina, Rio de Janeiro, Editora da Fiocruz.

Toledo Piza Jr., Salvador (1930), "Nouvelle théorie sur l'heredité", Boletim de Eugenia, 2 (23), pp. 4-5.

Toledo Piza Jr., Salvador (1932a), "O Casamento do branco com o preto à luz da Biologia”, Boletim de Eugenia, 4 (38), pp. 31-39.

Toledo Piza Jr., Salvador (1932b), "A hereditariedade da cor da pele no casamento branco-preto", Boletim de Eugenia, 4 (39), pp. 62-67.

Toledo Piza Jr., Salvador (1933a), “A Hereditariedade da Cor da Pele no Casamento Branco-Preto (Conclusão)", Boletim de Eugenia, 5 (41), pp. 5-12

Toledo Piza Jr., Salvador (1933b), "Um programa para a eugenia”, Boletim de Eugenia, 5, (42), pp. 16-17. 